\title{
Adjustable Module for Variable Depth Steel Arch Bridges
}

\author{
Y. Wang ${ }^{\mathrm{a}}$, A.P. Thrall ${ }^{\mathrm{a}, *}$, T.P. Zoli ${ }^{\mathrm{b}}$ \\ ${ }^{a}$ Kinetic Structures Laboratory, Department of Civil and Environmental Engineering and Earth Sciences, \\ University of Notre Dame, 159 Fitzpatrick Hall, \\ Notre Dame, IN 46556, USA \\ ${ }^{b}$ HNTB Corporation, 57th Floor, Empire State Building, 350 5th Ave, \\ New York, NY 10118, USA
}

\begin{abstract}
This paper presents a new strategy for modular steel construction: an adjustable module. Existing rapidly erectable, steel panelized bridge systems (e.g., Bailey, Acrow, MabeyJohnson) have limited material efficiency (span squared per weight) as they are comprised of rigid modules in a girder-type configuration. This paper addresses this limitation by developing a new adjustable, steel module comprised of a four-bar linkage which can form more efficient variable depth arch bridges. The objectives of this study are to (1) present methodologies for the development of rational variable depth three- and two-hinged arch forms, (2) evaluate the geometry of the adjustable module to scribe these rational forms, and (3) demonstrate the promise of the adjustable module through three-dimensional finite element analyses. Results show that a lightweight, adjustable module can form variable depth threeand two-hinged arches which increase the material efficiency by a factor of approximately 3 compared to existing systems. The module and other arch components can be comprised of American Institute of Steel Construction Steel Construction Manual standard rolled section sizes, thereby forming a versatile, kit-of-parts systems. The module weighs 1.41 times less than a conventional module, suggesting significant advancement compared to existing practice.
\end{abstract}

Keywords:

Modular construction, Arch bridges, Variable depth forms

\section{Introduction}

Advantages of modular construction (i.e., structures comprised of identical, prefabricated components) include reduced fabrication cost and high quality control of components. Existing modular or panelized steel bridging systems (e.g., Bailey, Acrow, Mabey-Johnson) have been widely used as rapidly erectable bridges for temporary construction in military and civilian (e.g., disaster relief) applications since World War II [1,2]. These systems feature

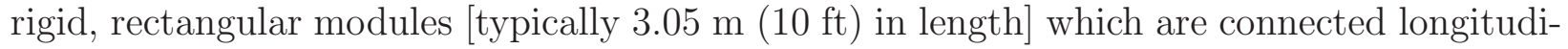
nally to form girder-type bridges (see for example the Bailey panel in Figure 1A). Versatility

\footnotetext{
${ }^{*}$ Corresponding author

Email addresses: ywang32@nd.edu (Y. Wang), athrall@nd.edu (A.P. Thrall), tzoli@hntb.com (T.P. Zoli)
} 
of these existing systems is achieved by stacking modules vertically and/or transversely to reach longer spans [up to $61.0 \mathrm{~m}$ to $91.4 \mathrm{~m}$ (200 to $300 \mathrm{ft}$ )] and/or higher load capacity (see for example the double-triple configuration - meaning two modules stacked transversely and three modules stacked vertically - of the Bailey system in Figure 1B). However, the material efficiency (quantified in this research as span squared per weight) of these longer, stacked configurations is limited since (1) material is placed at or near the neutral axis which contributes little to the strength of the system in a bending dominant girder configuration and

(2) stacking is not varied along the span despite varying moment and shear demands.

Prior work by the authors has demonstrated that longer spans and enhanced material efficiency can be achieved by arranging conventional, rigid modules in Pratt truss, bowstring truss, and network tied arch configurations [3]. However, these forms and the resulting material efficiency remain limited by the rigid module. For example, although the required structural depth of the network tied arch chord in this prior work is effectively zero at the hinges, the depth of the chord remains constant as it is formed by these rigid modules. This paper addresses this limitations by developing a new type of module which is capable of varying depth based on demand: an adjustable module which can form variable depth bridges.

\section{Research Significance}

While modular bridges comprised of rigid modules have been constructed since World War II, research on adjustable modules (i.e., modules that can change shape to generate variable depth forms) does not exist. This paper addresses this knowledge gap by presenting an adjustable module comprised of a four-bar linkage (i.e., a one kinematic degree of freedom mechanism comprised of four rigid links connected by four revolute joints, Figure 2). Here, the conventional, rigid module is re-imagined as a mechanism that can be adapted to scribe variable depth geometries. By numerically demonstrating the promise of this module and comparing it to existing panelized bridging strategies, this paper provides an impetus for future experimental research, and ultimately, for the technology transfer of this approach to government or industry.

\section{Objectives and Scope}

The aim of this research is to investigate the efficacy of an adjustable module (Figure 2) that can form variable depth bridges as a strategy for modular construction. Specific objectives toward this aim include: (1) presenting methodologies for the development of rational variable depth forms, (2) evaluating the geometry of the adjustable module to scribe these rational forms, and (3) demonstrating the promise of the adjustable module through three-dimensional finite element analyses. This paper first presents methodologies for determining rational variable depth forms for three- and two-hinged arches based on behavior under gravity loads. Then a scribing procedure, in which the adjustable module is shown to be able to generate these variable depth forms, is presented. With this scribing procedure, an exhaustive parametric study is performed to determine a module link length which features versatility in form (meaning that it is capable of scribing a large span range for both threeand two-hinged arches) and minimizes susceptibility to chord segment buckling (quantified 
as the longest unbraced length of an upper or lower chord segment squared to relate to Euler buckling). The promise of the adjustable module is then demonstrated through finite element analyses for a $91.4 \mathrm{~m}(300 \mathrm{ft})$ span. The material efficiency of the adjustable module for variable depth arch bridges is compared to an existing panelized system to demonstrate the advantages of this system compared to existing technology. This paper ultimately develops a strategy for panelized bridging with enhanced material efficiency and versatility of form.

\section{Methodologies for Development of Rational, Variable Depth Forms}

This section presents methodologies for the development of rational variable depth forms considered in this paper. The focus is on three- and two-hinged arches as arches utilize the cross-section more effectively than the flexural behavior of the girder configuration of existing panelized bridging systems. The depth of the arch can be varied based on demand by adjusting the module. Furthermore, the adjustable module (Figure 2) forms a naturally articulated pin for hinges in three- and two-hinged arch forms.

Three- and two-hinged arch bridges with flexible, lightweight decks are the arch forms for which a variable depth module can lead to greatest benefit. A flexible, lightweight deck is attractive for rapid erection of modular systems and offers advantages in transportability. This type of deck, however, leads to high bending in the arch under asymmetric live loading, particularly at the quarterpoint, requiring greater structural depth in these regions. At the hinges, effectively no structural depth is required, and the forms can narrow to the naturally articulated pins.

In the following sections, methodologies for developing rational variable depth three- and two-hinged arches are presented. Example forms are calculated for a $91.4 \mathrm{~m}$ (300 ft) span. The self-weight of the arch is assumed to be $5.25 \mathrm{kN} / \mathrm{m}(360 \mathrm{lbs} / \mathrm{ft})$. A lightweight deck is assumed to have a self-weight of $13.1 \mathrm{kN} / \mathrm{m}(900 \mathrm{lbs} / \mathrm{ft})$. The live load is taken as the distributed lane load prescribed by American Association of State and Highway Transportation Officials (AASHTO, hereafter) Load and Resistance Factor Design Specification [4] [9.34 $\mathrm{kN} / \mathrm{m}$ (640 lbs/ft)]. Load combinations include the self-weight (of both the arch and deck) with the live load acting over the entire span, over half the span on each side, over $5 / 8$ of the span on each side, and over $3 / 8$ of the center of the span to consider worst effect. It is assumed that two planes of arches will carry these loads, so the magnitude of each is divided in half.

\subsection{Three-hinged Arch}

A three-hinged arch form is desirable as the hinge at the crown enables the arch to adjust for thermal contraction/expansion and for settlement of the supports without imparting internal forces in the arch. This is particularly appealing for rapidly erected bridges for which foundation conditions may be unknown or undesirable.

A rational form for a variable depth three-hinged arch can be developed using graphic statics. Graphic statics is a graphical analysis and design tool for truss-type (i.e., axial load bearing only) structures. While this method has been used for centuries, it is only recently gaining greater attention in the structural engineering research community (e.g., [5-7]). This method requires only drafting tools (computerized drafting software packages, e.g., AutoCAD [8], are often used today for increased accuracy and convenience). Given 
a loading - in this case distributed loads discussed above which are approximated as point

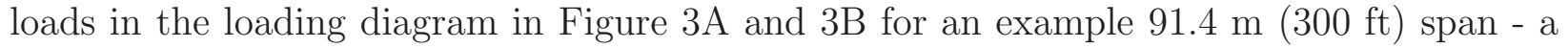
"form diagram" can be developed which represents the positioning of structural members for which only axial load (i.e., compression for the arches discussed here) is carried. A reciprocal "force diagram" represents the magnitude of the force in each member under that loading. Rays of the force diagram are parallel to the structural members in the form diagram. The upper-case letters on the loading diagram label intervals between externally applied loads. These correspond to points in the force diagram, identified there by the same, but lower-case letters. Structural members in the form diagram are labeled by the two lower-case letters of the corresponding force ray [9-11]. To generate the shape of the three-hinged arch under a uniformly distributed load in this research (Figure 3A, for self-weight and live load over the full span), the load line (vertical line a-r) in the force diagram is first drawn. The length of line between each lowercase letter corresponds to the scaled magnitude of load applied in the interval between the corresponding upper-case letters in the loading diagram. Under a uniformly distributed load on a symmetric structure, the middle segment (oi) of the form diagram is horizontal. As the form and force diagrams are reciprocal, the corresponding force ray (oi) must also be horizontal. On the force diagram, the point o corresponds to the "pole" at which all rays must meet for this system. While the length of ray oi is not yet known, point o must fall somewhere along this horizontal ray. As the ideal (i.e., no bending) shape of an arch under a uniformly distributed load is a parabola, the angle of the last segments of the form diagram oa and oq can be determined based on the geometry of a parabola given a desired span and span-to-rise ratio. Corresponding parallel rays can be drawn on the force diagram, thereby locating pole o. The remaining rays on the force diagram can then be drawn, connecting each remaining lowercase letter b-q to the pole o. Finally, the form diagram can be completed by drawing line segments parallel to those in the force diagram. A similar process can be carried out for all loading scenarios discussed above, to develop form diagrams, also known as "pressure lines," for each load (see for example Figure 3B for dead load and live load over left half the span). For these other loadings, the end segment angles in the form diagram are no longer required to relate to a parabolic shape. Instead, it is required that each form diagram cross through the hinge at the crown of the form diagram for the uniformly distributed load (i.e., dead load and live load over the full span, Figure 3A) $[11]$.

In this research, graphic statics was used to develop the pressure lines under all of the different loading scenarios discussed above (gray lines in Figure 4). These lines were calculated at discrete points (in this case 19 discrete points) as the distributed loads must be discretized to perform the graphic statics procedure. A black line in Figure 4 indicates the envelope of these lines. The envelope of pressure lines provides the form for a three-hinged arch such that the arch is always in compression [11]. It is therefore appealing to have the upper and lower chord follow the geometry of the envelope of the pressure lines to eliminate stress reversals in the chord and the resulting susceptibility to fatigue and fracture. Furthermore, connection design between chords is simplified if the chords are only subjected to compression and these connections would likewise not be susceptible to fatigue and fracture. In any kind of modular or panelized system, a minimum depth is required. To account for this, an additional $1.52 \mathrm{~m}(5 \mathrm{ft})$ of depth is added to the discrete points of the upper pressure line envelope. Polynomial curves are fit to the upper and lower pressure line envelope 
discrete points to generate continuous functions between which the adjustable module can be scribed. The upper and lower chord would then be generated by connecting straight line segments between the module revolute joints.

Figure 5 shows the coordinates of the discrete points from the envelope of pressure lines [gray squares, with additional $1.52 \mathrm{~m}$ (5 ft) of depth added], the continuous polynomial curves fit to these points (gray lines), and the module scribed within these curves (black circles represent revolute joints, black lines represent links) for an example $91.4 \mathrm{~m} \mathrm{(300 \textrm {ft } )}$ span with a span-to-rise ratio of 5 . Figure $6 \mathrm{~A}$ shows the elevation and plan view of the final form for the example three-hinged arch.

\subsection{Two-hinged Arch}

Alternatively, a two-hinged arch can be desirable over a three-hinged arch as the hinge at the crown can be avoided, thereby leading to savings in fabrication cost. Furthermore, the redundancy of the two-hinged arch enables the system to maintain stability even if a chord segment is damaged as it is capable of redistributing moment. Since the two-hinged arch form is statically indeterminate, an alternative approach to developing its form based on moment demand under asymmetric live load and in-plane buckling of the arch is used.

The bending moment under asymmetric live loads of this statically indeterminate structure can be determined by first finding the horizontal reaction using the method of virtual work. In the method of virtual work, the horizontal degree of freedom at one of the arch hinges is hypothetically released, thereby enabling horizontal translation of the arch under load. This horizontal translation due to load is determined mathematically. Likewise, the horizontal translation due to only a horizontal thrust is determined. These are set equal to one another and the horizontal thrust (i.e., reaction) is therefore found. More specifically in this research, the centerline of the arch, with a span $(S)$ and rise $(D)$, is chosen to be a parabola given by the following equation:

$$
y=-\frac{4 D}{S^{2}} x^{2}+D
$$

as this is the ideal form for an arch carrying only compression under a uniformly distributed load (see Figure 7 for the assumed coordinate system, in which $x$ is the horizontal coordinate and $y$ is the vertical coordinate). Assuming that shear and axial deflections are negligible, the horizontal reaction $(H)$ is then:

$$
H=\frac{\int_{0}^{s} \frac{M_{o} y d s}{E I}}{\int_{0}^{s} \frac{y^{2} d s}{E I}}
$$

where $M_{o}$ is the bending moment (if the horizontal reaction is released), $s$ is the length along the arch centerline, $E$ is the modulus of elasticity, and $I$ is the moment of inertia [12]. These integrals can be approximated as summations over curved segments of the arch (in this case, 16 curved segments were considered along the full span). With the horizontal reaction determined, the axial force, shear, and bending moment can then be calculated using static equilibrium conditions along the full length of the arch.

Further, deflections induced by asymmetric live loads in combination with axial forces

from self-weight and the live load cause increased deflection and moment in the arch [13]. The 
increased deflection and moment can be accounted for in design by moment magnification factors $\left(A_{F S}\right)$, as follows:

$$
A_{F S}=\frac{1}{1-\frac{P_{4}}{a_{4} F_{E}}}
$$

where $P$ is the axial force in the arch at the quarterpoint (subscript 4), $a$ is the cross-sectional area, and $F_{E}$ is the Euler buckling stress which can be calculated by:

$$
F_{E}=\frac{\pi^{2} E}{\left(\frac{k C}{r_{4}}\right)^{2}}
$$

where $C$ is half of the length of the arch, $r$ is the radius of gyration, and $k$ is an effective length factor based on the arch restraint (for 2-hinged arches with a span-to-rise ratio of 5, this is 1.10) [13]. Furthermore, arches are susceptible to in-plane buckling, also related to the Euler buckling stress $F_{E}$. Therefore, the strategy for a rational variable depth two-hinged arch form is determined based on the Euler buckling stress and the moment demand under live load.

In this research, the depth of the arch is related to the Euler buckling stress and the moment demand as follows. The cross-section of the arch is defined as shown in Figure 8, where $a$ refers to the cross-sectional area of the upper chord and the lower chord and $d$ is the distance between the chords. It is assumed that the cross-sectional area of the chords remains constant over the arch, but the depth $d$ varies. The moment of inertia of the arch is approximated as $I=a d^{2} / 2$ (i.e., the moment of inertia of each chord about its own centroid is negligible, any contribution from the adjustable module is negligible), with a radius of gyration $r=d / 2$. The flexural stress $\sigma$ at the center of each chord due to a moment $M$ is:

$$
\sigma=\frac{M}{a d}
$$

The smallest cross-sectional area that would result in yielding of the section by flexure is therefore:

$$
a=\frac{M}{\frac{F_{y}}{\Omega} d}
$$

where $M$ is the largest magnitude of moment under all load combinations, $F_{y}$ is the yield strength [the chords are assumed to be A992 steel with a yield strength of $F_{y}=345 \mathrm{MPa}$ (50 $\mathrm{ksi}$ )], and $\Omega$ is a safety factor (taken to be 1.67 as this is the safety factor for compression elements in Allowable Stress Design [14]). Requiring that the axial stress (from the axial force and the cross-sectional area) be less than the Euler buckling stress at the quarterpoint, the following relationship is determined:

$$
\frac{P_{4}}{\frac{M}{\frac{F_{y}}{\Omega} d_{4}}} \leq \frac{\pi^{2} d_{4}^{2} E}{4(k L)^{2}}
$$

and therefore the depth $d_{4}$ at the quarterpoint is: 


$$
d_{4} \geq \frac{4 P_{4} \frac{F_{y}}{\Omega}(k L)^{2}}{M \pi^{2} E}
$$

The minimum cross-sectional area is then determined using Equation 6 with this depth at the quarterpoint and the corresponding moment (maximum magnitude over all load combination) at the quarterpoint. The depth throughout the arch is then found by:

$$
d \geq \frac{M}{a \frac{F_{y}}{\Omega}}
$$

where $M$ is the moment (maximum magnitude over all load combination) along the arch at which $d$ is calculated. Figure 9 shows the moment for all load combinations (in gray) and

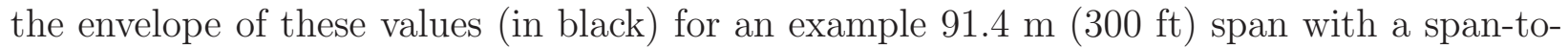
rise ratio of 5. The depth is calculated at discrete points (in this case 19 discrete points)

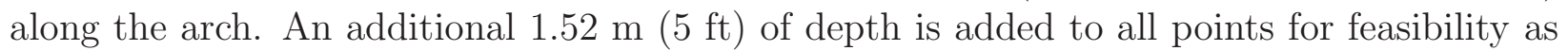
discussed for the three-hinged arch. Polynomial curves are fit to the resulting data points to arrive at continuous curves between which the module can be scribed. The upper and lower chord would then be generated by connecting straight line segments between the module revolute joints.

Figure 10 shows the coordinates of the discrete points from the depth calculation [gray squares, with additional $1.52 \mathrm{~m}$ ( $5 \mathrm{ft}$ ) of depth added], the continuous polynomial curves fit to these points (gray lines), and the module scribed within these curves (black circles represent revolute joints, black lines represent links) for an example $91.4 \mathrm{~m}(300 \mathrm{ft}$ ) span with a span-to-rise ratio of 5 . Figure $6 \mathrm{~B}$ shows the elevation and plan view of the final form for the example two-hinged arch.

\section{Evaluation of Geometry of Adjustable Module}

The prior sections developed the rational forms for three- and two-hinged arches (given the span $S$ and the span-to-rise ratio) based on governing load demands. This form development culminates in upper and lower continuous curves that serve as bounds for variable depth arches. This paper proposes that these variable depth arches can be formed through modules comprised of four-bar linkages with a link length $l$ (Figure 2).

Linkages have been widely studied since the 18th century $[15,16]$, with applications largely concentrated in mechanical engineering and few examples in civil engineering. In movable bridge engineering, linkages have been implemented for the rotation of overhead counterweights in Strauss heel-trunnion bascules [17] and have been suggested as both the load-bearing and kinematic elements of bascule bridges [18]. Linkages have also been investigated for rapidly deployable bridges $[19,20]$. The novelty of the research presented here lies in the use of linkages to develop variable depth forms within a framework of modular construction.

Given the topology of the adjustable module shown in Figure 2, this section evaluates the ability of this geometry to scribe the rational, variable depth forms discussed in the previous section. First, this section presents the geometric process by which these modules scribe variable depth forms. Then a parametric study is performed to determine an optimized link length. 


\subsection{Geometric Process for Scribing Adjustable Module}

The modules are scribed between the upper and lower continuous curves of the rational forms by determining the intersection of circles $(D)$ with radius of the link length $l$ and these continuous curves (Figure 11). More specifically, the top corner $(A)$ of the module intersects with the upper curve of the rational form and the bottom corner $(B)$ with the bottom curve. The other corners $(C)$ connect modules to one another (Figure 2 and 11). The upper chord is then formed by connecting the $A$ points to one another by straight line segments, and the lower chord is likewise found by connecting the $B$ points (black dotted lines in Figure 11). For the scribing procedure, a desired span must initially be specified. However, it is unlikely that a module would end exactly at the desired span. Furthermore, it is advantageous to end the scribing procedure at a corner $C$ as this forms an articulated pin for the hinged arches. Therefore, a scribing termination criteria that a point $C$ be within $2 l$ of the desired span end has been implemented for the scribing procedure. As a result the actual span will be slightly less than the desired span. It is also required that there be at least a 5 degree angle between links for feasibility.

\subsubsection{Three-hinged Arch}

To achieve a hinge at the crown of the three-hinged arch (Figure 11A), the modules begin at a point $C_{1}$ at the crown with a horizontal coordinate $C_{1 x}=0$ and a vertical coordinate $C_{1 y}$ which is the average value of the vertical coordinates of the upper and lower curves when $x=0$. The vertical coordinates of $A_{1}$ and $B_{1}$ are then found by the intersection of the upper and lower curves, respectively with circle $D_{C 1}$ - centered at $C_{1}$ with a radius of link length $l$ - which has the following equation:

$$
\text { Circle } D_{C 1}: x^{2}+\left(y-C_{1 y}\right)^{2}=l^{2}
$$

(Figure 11A, first row). The next point $C_{2}$ is found by the intersection of circle $D_{A 1}$ with radius $l$ centered at point $A_{1}$ with the equation:

$$
\text { Circle } D_{A 1}:\left(x-A_{1 x}\right)^{2}+\left(y-A_{1 y}\right)^{2}=l^{2}
$$

and circle $D_{B 1}$ with radius $l$ centered at point $B_{1}$ with the equation:

$$
\text { Circle } D_{B 1}:\left(x-B_{1 x}\right)^{2}+\left(y-B_{1 y}\right)^{2}=l^{2}
$$

(Figure 11A, second row). The following points $A_{2}$ and $B_{2}$ are found by the intersection of circle $D_{C 2}$ with radius $l$ centered at $C_{2}$ with the equation:

$$
\text { Circle } D_{C 2}:\left(x-C_{2 x}\right)^{2}+\left(y-C_{2 y}\right)^{2}=l^{2}
$$

and the upper and lower curves, respectively (Figure 11A, third row). This continues until the termination at the springing when a point $C$ is within $2 l$ of the desired span (Figure 11A, fourth row). The upper and lower chords are ultimately formed by connecting the $A$ points to one another by straight line segments and by connecting the $B$ points (black dotted lines in Figure 11A, fourth row). Note that at the crown and the springing, the last links of the module form segments of the upper and lower chord. 
Given the span $S$, the span-to-rise ratio, the loads, and the link length, the full geometry of the arch can then be determined for the three-hinged arch. See Figure 5 for an example of the module scribed onto the rational form for a span of $91.4 \mathrm{~m}$ (300 ft), a span-to-rise ratio of 5 , and a link length $l=3.05 \mathrm{~m}(10 \mathrm{ft})$.

\subsubsection{Two-hinged Arch}

Scribing for the two-hinged arch is achieved using the same approach (Figure 11B). However, a structural depth is achieved at the crown by beginning mid-module, meaning that points $A_{1}$ and $B_{1}$ of the module are at midspan. The horizontal coordinates of these points are: $A_{1 x}=0$ and $B_{1 x}=0$ and the vertical coordinates are that of the upper and lower curves at $x=0$, respectively. The coordinates of the point $C_{1}$ are found by the intersection of circle $D_{A 1}$ with a radius $l$ centered at $A_{1}$ with the equation:

$$
\text { Circle } D_{A 1}: x^{2}+\left(y-A_{1 y}\right)^{2}
$$

with circle $D_{B 1}$ with a radius $l$ centered at $B_{1}$ with the equation:

$$
\text { Circle } D_{B 1}: x^{2}+\left(y-B_{1 y}\right)^{2}
$$

(Figure 11B, first row). The coordinates of $A_{2}$ and $B_{2}$ are then found by the intersection of upper and lower curves with circle $D_{C 1}$ which has the following equation:

$$
\text { Circle } D_{C 1}:\left(x-C_{1 x}\right)^{2}+\left(y-C_{1 y}\right)^{2}=l^{2}
$$

(Figure 11B, second row). The coordinates of $C_{2}$ are found by the intersection of circle $D_{A 2}$ (centered at $A_{2}$ with radius $l$ ):

$$
\text { Circle } D_{A 2}:\left(x-A_{2 x}\right)^{2}+\left(y-A_{2 y}\right)^{2}=l^{2}
$$

with circle $D_{B 2}$ (centered at $B_{2}$ with radius $\left.l\right)$ :

$$
\text { Circle } D_{B 2}:\left(x-B_{2 x}\right)^{2}+\left(y-B_{2 y}\right)^{2}=l^{2}
$$

(Figure 11B, third row). This process continues until the termination criteria (i.e., a point $C$ is within $2 l$ of the desired span) is met (Figure 11B, fourth row). As for the case of the three-hinged arch, the upper and lower chords are formed by connecting the $A$ points to one another by straight line segments and by connecting the $B$ points (black dotted lines in Figure 11B, fourth row). At the springing, the last links of the module form segments of the upper and lower chord.

See Figure 10 for an example of the module scribed onto the rational form for a span of $91.4 \mathrm{~m}(300 \mathrm{ft})$, a span-to-rise ratio of 5 , and a link length $l=3.05 \mathrm{~m}(10 \mathrm{ft})$.

\subsection{Parametric Investigation to Determine Optimized Link Length}

Parametric studies were performed to determine an optimized link length which is feasible for a wide variety of span lengths and for which the arch chord segments have a low susceptibility to in-plane buckling. More specifically, the form development and scribing procedures discussed above were carried out for a range of link lengths [from 1.52 to $4.57 \mathrm{~m}$ (5 to $15 \mathrm{ft}$ ) in increments of $0.305 \mathrm{~m}(1 \mathrm{ft})$ ] and spans [considering spans from $61.0 \mathrm{~m}$ to $183 \mathrm{~m}(200$ to 
$600 \mathrm{ft})$ in increments of $15.2 \mathrm{~m}(50 \mathrm{ft})$, all with a span-to-rise ratio of 5]. Some combinations are not feasible as the link lengths are too short to scribe the rational forms. Since arches are particularly susceptible to in-plane buckling (even more so than out-of-plane buckling as lateral bracing can be provided to prevent out-of-plane buckling modes) [13], this parametric study investigated a metric related to this behavior. More specifically, the longest in-plane unbraced length of the upper or lower arch chord $(L$ in Figure 11, connecting points $A$ or points $B$ ) was found through the scribing procedure for each link length and span combination. The metric used here considers this in-plane braced length squared $\left(L^{2}\right)$ to relate to the critical Euler buckling load. The highest possible value for this metric is $(2 l)^{2}$.

The results of the parametric study to determine the link length for the three- and twohinged arches are shown in Figure 12 and Figure 13, respectively. Note that the span lengths shown are not in even increments due to the module scribing termination criteria discussed earlier. The spans shown here are the horizontal distances from springing to springing (from a point $C$ to a point $C$ on opposite sides of the arch). From both Figure 12 and 13, it is clear that the smallest link length which is capable of achieving the full range of considered spans for three- and two-hinged arches is $3.05 \mathrm{~m}(10 \mathrm{ft})$. As expected, this link length also relates to the lowest value of $L^{2}$.

A link length of $3.05 \mathrm{~m}$ (10 ft) is also supported by precedents in panelized bridging systems. The Bailey, Acrow, Mabey-Johnson panelized systems all use modules that are $3.05 \mathrm{~m}$ (10 ft) long [1, 2], indicating that this is a reasonable size for handling rapidly erectable bridge components. Transportation advantages of this link length include that the module can be transported flat with a total length of $6.10 \mathrm{~m}(20 \mathrm{ft})$. This would make the module transportable in $6.10 \mathrm{~m}(20 \mathrm{ft}$ ) or $12.2 \mathrm{~m}$ (40 ft) ISO containers. Note that this length is approximate since, in reality, the module would not be able to collapse entirely on itself. Furthermore, there is an advantage in using the least number of modules to achieve desired spans as this reduces the number of connections required. This reduces the field erection time and also the cost of the construction. By choosing the longest link length which is easily transportable, additional savings could be realized.

Based on the parametric study related to form, precedents in panelized bridging systems

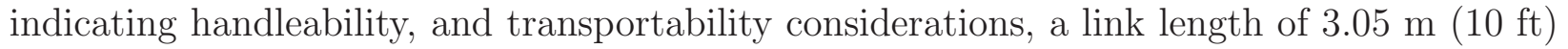
was selected for further study (Figure 2).

\section{Demonstration of the Promise of the Adjustable Module}

To demonstrate the promise of the module for variable depth three- and two-hinged

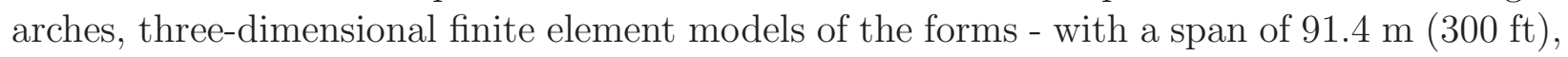
a span-to-rise ratio of 5 , and a link length $l=3.05 \mathrm{~m}$ (10 ft) developed in the prior sections - were built and analyzed under combined dead, live, and wind loads (Figure 6). The dead load includes the self-weight of the arch members as well as a superimposed dead load for the deck $[13.1 \mathrm{kN} / \mathrm{m}(900 \mathrm{lbs} / \mathrm{ft})]$. As considered earlier, the live load is the distributed lane load prescribed by AASHTO $[9.34 \mathrm{kN} / \mathrm{m}(640 \mathrm{lbs} / \mathrm{ft})[4]]$, which is considered to act over (1) the full span, (2) half the span, (3) 5/8 of the span, and (4) 3/8 of the center of the span (i.e., loads to generate worst effect on the arch). Projected gravity loads are applied to the upper chords (half of this load on each plane). An assumed $2.39 \mathrm{kPa}$ (50 psf) wind load is applied laterally to one plane of the arch. Linear (eigenvalue) buckling analyses of the forms 
were performed under these load combinations to understand global behavior of the system. More specifically, the software package SAP 2000 (v.17.3.0) was used to solve the following problem:

$$
[K-\lambda g(p)] \Psi=0
$$

where $K$ is the stiffness matrix, $\lambda$ is the eigenvalue matrix, $g$ is the geometric stiffness for loads $p$, and $\Psi$ is the eigenvector matrix [21]. Section sizes and a lateral bracing scheme were designed to achieve buckling factors (meaning the factor by which the load would need to be multiplied by to cause buckling) that exceed 2.5 for all load combinations. Only service loads were considered (i.e., load factors of 1 ) for this preliminary analysis.

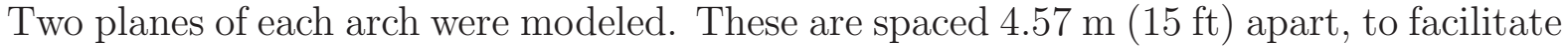
a $3.66 \mathrm{~m}$ (12 ft) design lane load as per AASHTO [4]. The springing of each arch plane is restrained to prevent translation in all directions and permit rotation only about the axis perpendicular to the plane of the arch. Arch chords are comprised of straight line segments. Connections between these straight line segments are moment-resisting, with the exception of the chords at the crown of the three-hinged arch and at the springing for both arches where in-plane rotation is permitted to achieve hinges. The chords are wide flange W10X39 sections [14], oriented so that the strong axis is in the plane of the arch to resist out-of-plane buckling. The module members, L5X5X5/16 angle sections [14], are pin-connected to one another and to the arch in the plane of the arch. This is to simulate the revolute joint required for the modules to be adjustable. Symmetric angle sections were chosen to resist in- and out- of plane buckling. Chevron-type lateral bracing connects the planes. The same section sizes are used for the braces as for the chord to minimize the number of different types of sections in a rapidly erectable environment. These braces intersect the arch chord at each segment midpoint to avoid connecting to the chord at the same location as the module. All brace connections are moment-resisting. These section sizes were selected using an iterative approach in which the smallest section sizes (i.e., lowest weight) were chosen to achieve the desired buckling factors. A premium was placed on reducing the self-weight of the module to ensure handleability and transportability. The same American Institute of Steel Construction (AISC) Steel Construction Manual [14] standard rolled section sizes were chosen for the three- and two-hinged arch schemes to culminate in a unified kit-ofparts system which could be used for either form based on the site constraints and desired performance.

Figure 14 shows the buckled shape of these three- and two-hinged arches for the most critical loading in isometric, plan, and elevation views. The buckling mode for each system is local in-plane buckling of the chord under dead, asymmetric live, and wind loads. In plan view (second row), negligible out-of-plane buckling is observed.

\section{Discussion}

To understand the advantages of the approach to modular construction presented here, the variable depth three- and two-hinged adjustable module arch forms must be compared to an existing panelized bridging system in a girder-type configuration. The Bailey Bridge [22], in a triple-triple configuration to achieve the longest spanning simply supported bridge [64.0 $\mathrm{m}(210 \mathrm{ft})]$ allowable, is chosen as a representative existing system for comparison. 
These systems are compared using a material efficiency metric - defined to be the span length squared divided by the self-weight because the moment of a simply supported beam under a uniform load is proportional to the span squared. This metric was chosen as a means of comparing different span systems and has been used in prior work related to panelized bridging systems [3]. Note that the self-weight does not include the deck. The Bailey Bridge weight is based only on the weight of the panels and does not include the weight of the lateral bracing for simplicity. The weight of a module is also compared as this relates to the handleability and transportability of the system. For reference, a single Bailey panel weighs $2.57 \mathrm{kN}(577 \mathrm{lbs}$ ) and can be carried by just 6 soldiers when using carrying bars [22]. Table 1 provides the data related to the self-weight and material efficiencies of the different forms considered.

The adjustable module is 1.4 times lighter than the Bailey panel. This indicates the adjustable module's handleability and transportability as it would be capable of being carried by less than 6 soldiers. Further weight reductions may also be possible if the adjustable module were to be comprised of aluminum or advanced composites.

The material efficiency of both the three- and two-hinged arch bridges far exceeds that of the Bailey Bridge (by roughly a factor of 3). Further weight reductions (and therefore increases in material efficiency) for the three- and two-hinged arches may also be possible if the section sizes of the lateral bracing is reduced. These were chosen to be the same as the chord to result in a unified kit-of-parts system with the fewest number of different section sizes. Ultimately, this comparison shows the advantages of variable depth arches comprised of adjustable modules.

These preliminary studies have shown the promise of this new strategy for modular construction for the specific cases of variable depth three- and two-hinged arches. The adjustable module could be used to form other bridge types where variable depths can provide material efficiency advantages, such as continuous trusses. It could also be used to develop constant depth curved geometries which are typically difficult to construct.

\section{Conclusion}

This paper investigated the efficacy of an adjustable module as a strategy for modular bridging. This strategy improves upon the material inefficiencies of existing panelized bridge systems comprised of rigid modules in a girder-type configuration by (1) forming arches which more efficiently use the available cross-section in compression as opposed to flexural behavior, and (2) facilitating a variable depth form based on demand to reduce system weight. This enhanced material efficiency is desirable for rapidly erectable, temporary systems where transportability and handelability are at a premium. These systems could be realized for military operations, to restore vital lifelines following natural or anthropogenic hazards, or as an accelerated bridge construction approach for civil applications.

This research presented methodologies for the development of rational variable depth three- and two-hinged arch forms based on behavior under gravity loads. Then the geometry of the adjustable module to scribe these rational forms was evaluated. This included an exhaustive parametric study to determine an optimized link length to achieve diversity in achievable span lengths and limit susceptibility of the arch chord segments to in-plane buckling. The promise of the adjustable module was demonstrated for example $91.4 \mathrm{~m}$ (300 
ft) span three- and two-hinged arches. Preliminary designs of the forms through threedimensional finite element analyses led to the determination of section sizes and a lateral bracing scheme for both forms, ultimately culminating in a kit-of-parts system that can form variable depth three- or two-hinged arches.

The material efficiency of the adjustable module for variable depth arch bridges was compared to an existing panelized system (i.e., Bailey Bridge). Results show that a lightweight, adjustable module can form variable depth three- and two-hinged arches which increase the material efficiency by a factor of approximately 3 compared to existing systems. Furthermore, the module weighs 1.41 times less than a conventional module, suggesting significant advancement compared to existing practice.

This research numerically investigated the efficacy of an adjustable module. This investigation provides an impetus for future experimental research in which the behavior of an adjustable module would be further studied and understood. To ultimately transfer this technology to government or industry, further detailed numerical research is also needed. To demonstrate the promise of the adjustable module, the linear (eigenvalue) buckling analyses presented in this paper evaluated the global behavior of the variable depth arches. However, individual components of the systems must be designed for strength, extreme event, service, and fatigue limit states as required by AASHTO. Furthermore, to investigate the effect of manufacturing imperfections, (geometrically) nonlinear buckling analyses should be performed. An effective substructure strategy, capable of resolving the horizontal thrust of the arches as well as the vertical reactions, must be developed. The design of a tie could also be considered as opposed to resolving the horizontal thrust into the substructure. It is envisioned that the revolute joint connecting the module links would be a bolt which is tightened on site. Detailed analysis and experimental investigation of this connection detail is required. Durability and fatigue life of the module and its revolute joint should be evaluated.

\section{Acknowledgments}

This material is based upon work supported by the National Science Foundation under Grant No. CMMI-1351272. The support of Program Managers Drs. Kishor Mehta and Y. Grace Hsuan is gratefully acknowledged. 


\begin{tabular}{|l|c|c|c|c|}
\hline Bridge Form & Mod. Wt. $(k N)$ & No. of Mod. & Wt. $(k N)$ & Mat. Eff. $\left(m^{2} / k N\right)$ \\
\hline Var. Depth Three-hinged & 1.83 & 36 & 592 & 13.7 \\
Var. Depth Two-hinged & 1.83 & 34 & 590 & 12.2 \\
Bailey Bridge System & 2.57 & 378 & 970 & 4.22 \\
\hline
\end{tabular}

Table 1: Material efficiency for variable depth three- and two-hinged arch bridges, and an existing panelized systems (i.e., Bailey Bridge). Abbreviations: Mod. = module, Wt. = weight, No. = number, Mat. = material, Eff. =efficiency. 

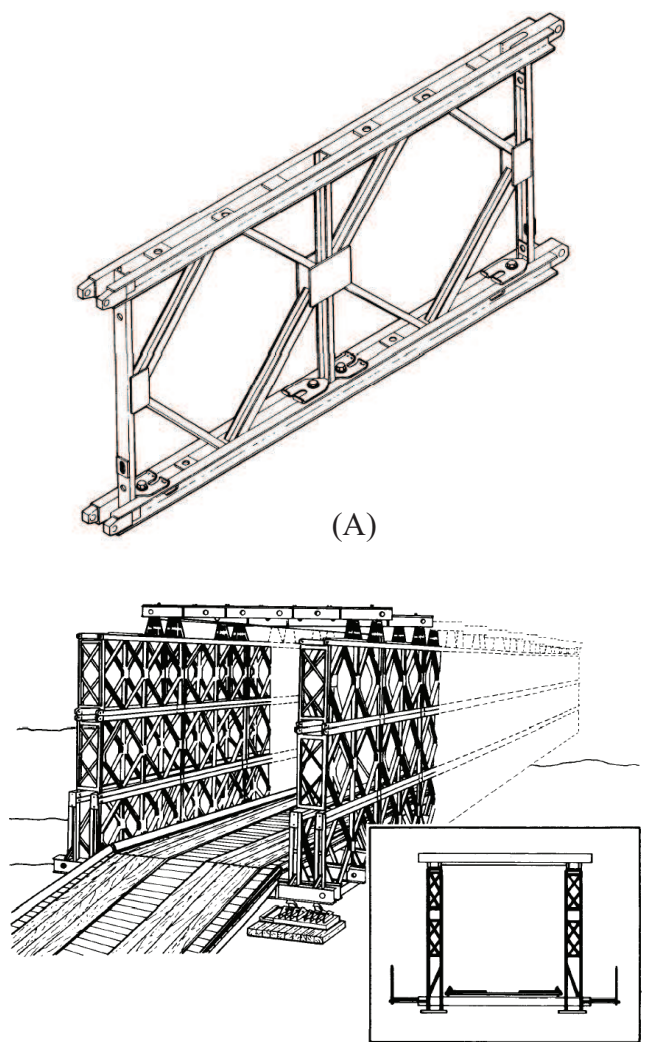

(B)

Figure 1: Bailey Bridge panel (A) shown in a double-triple girder-type configuration (B). Images courtesy of the US Army [22]. 


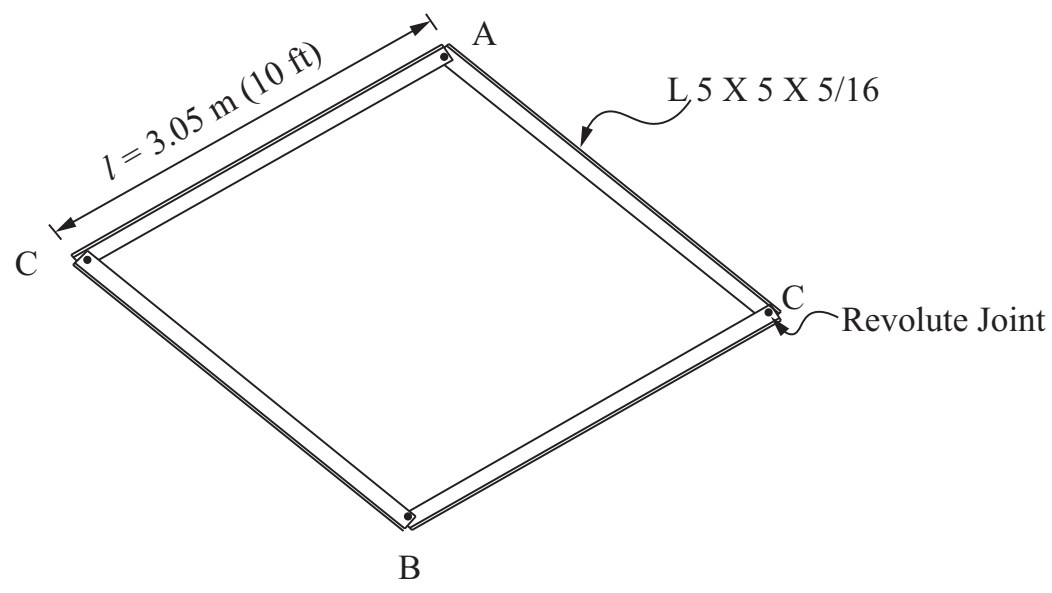

Figure 2: Elevation of adjustable module, including the optimized link length $l$ determined through the exhaustive parametric study and the section sizes selected through design and analysis. 
Loading Diagram
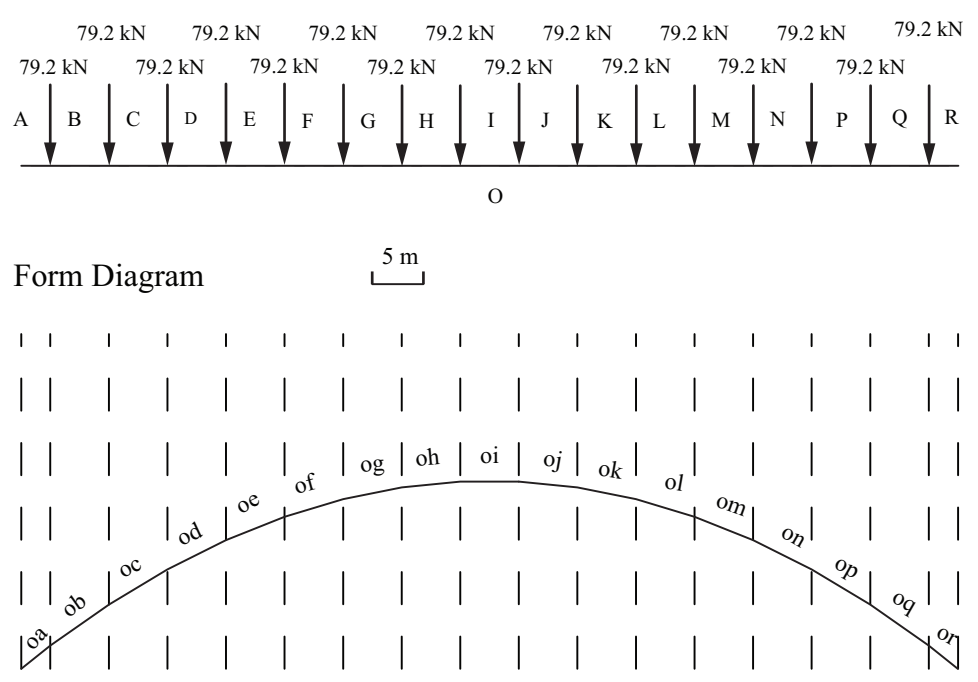

(A)

Loading Diagram

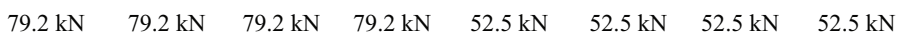

$$
\begin{aligned}
& \begin{array}{llllllll}
79.2 \mathrm{kN} & 79.2 \mathrm{kN} & 79.2 \mathrm{kN} & 79.2 \mathrm{kN} & 52.5 \mathrm{kN} & 52.5 \mathrm{kN} & 52.5 \mathrm{kN} & 52.5 \mathrm{kN}
\end{array}
\end{aligned}
$$

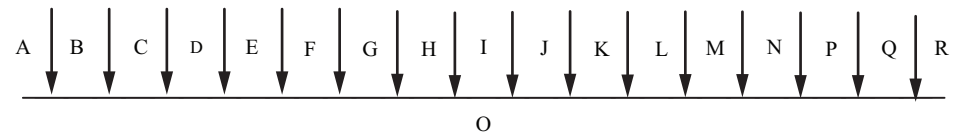

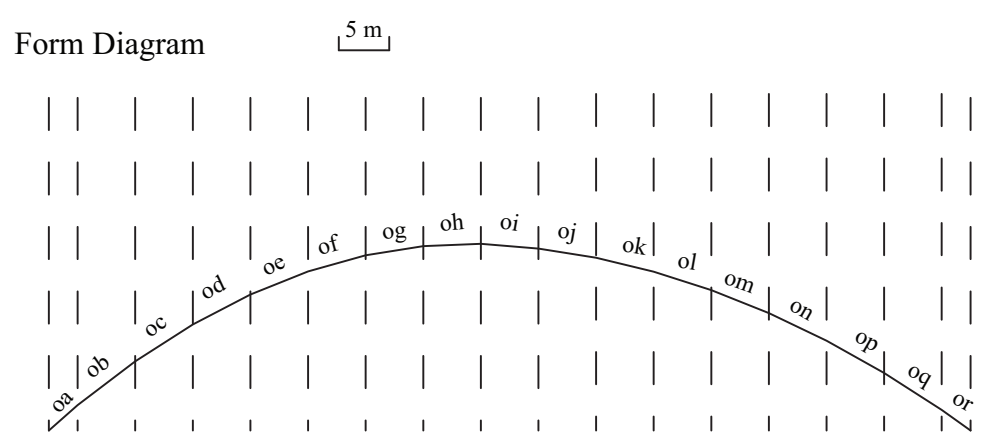

(B)
Force Diagram

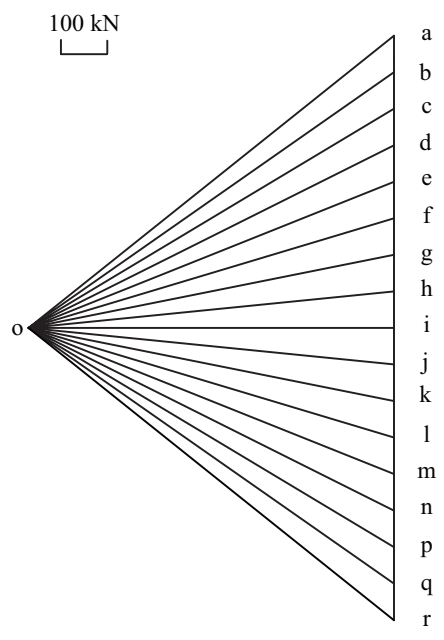

Force Diagram

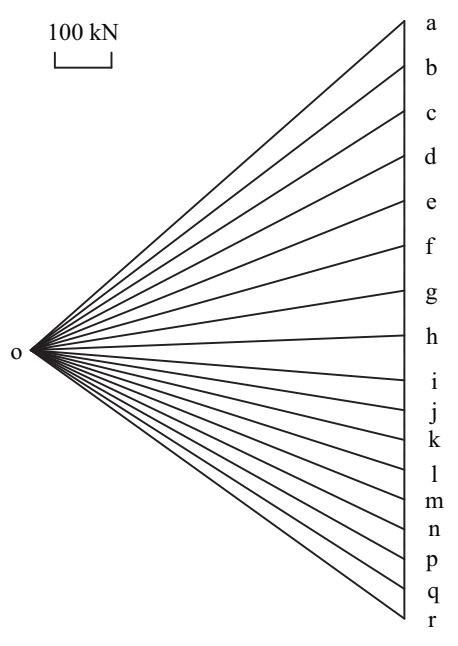

Figure 3: Graphic statics calculation for $91.4 \mathrm{~m}(300 \mathrm{ft})$ three-hinged arch with a span-to-rise ratio of 5 subject to (A) dead load and live load over full span and (B) dead load and live load over left half of span. 


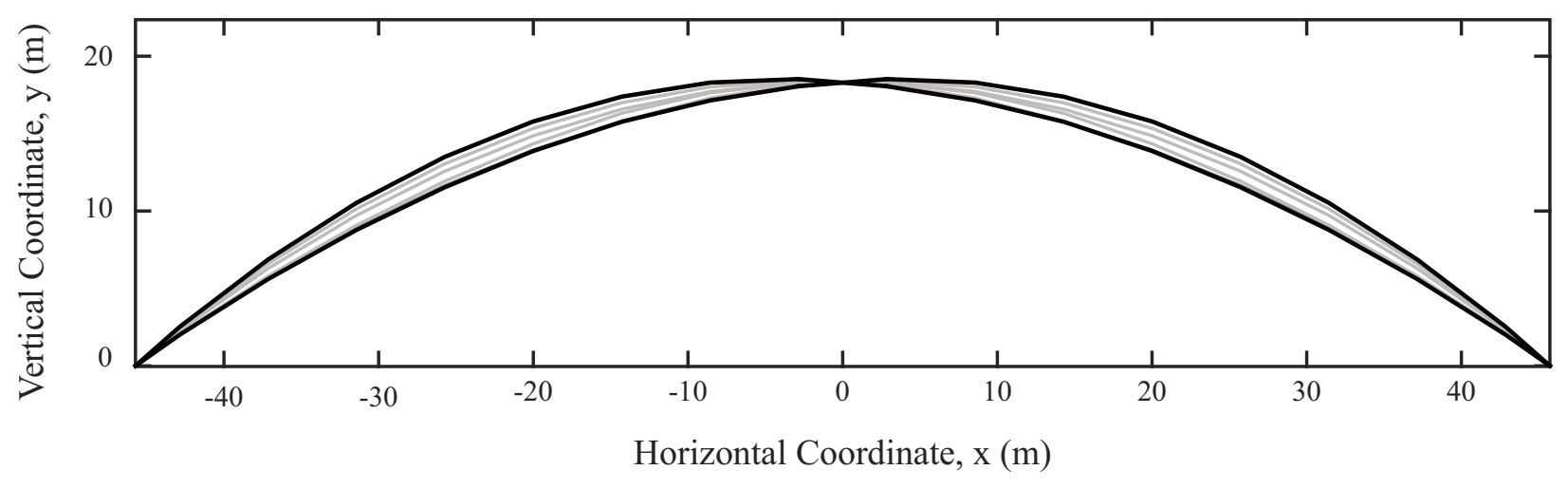

Figure 4: Graphic statics pressure lines for $91.4 \mathrm{~m}(300 \mathrm{ft})$ three-hinged arch with a span-to-rise ratio of 5. Envelope is shown in black. Individual pressure lines for different load combinations shown in gray. 


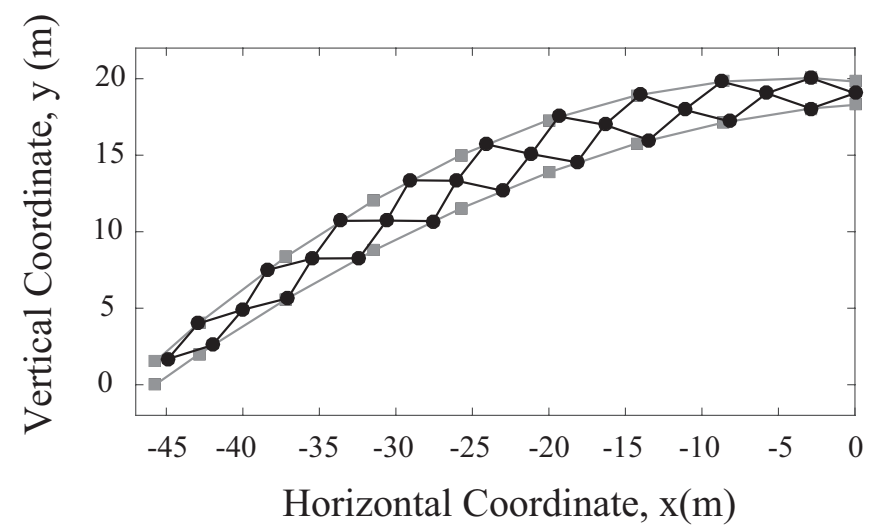

Figure 5: Form development for variable depth three-hinged arch. Gray square markers indicate the discrete points at which the envelope of pressure lines was calculated. The polynomial curves fit to these points are shown as gray lines. The module links are shown as black lines with the revolute joints as black circles. Shown for $91.4 \mathrm{~m}(300 \mathrm{ft})$ span, span-to-rise ratio of 5 . 

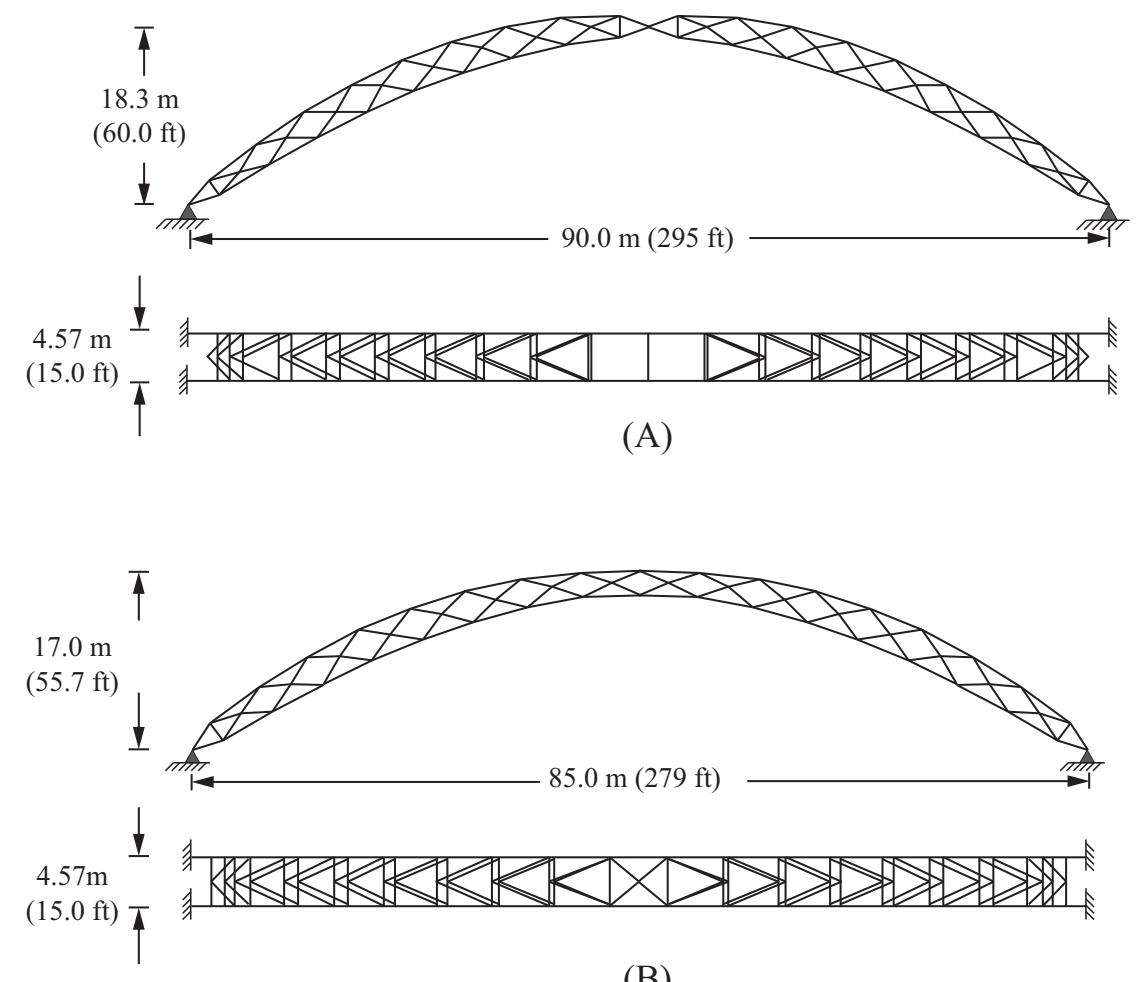

(B)

Figure 6: Elevation and plan views of (A) three-hinged arch and (B) two-hinged arch. 


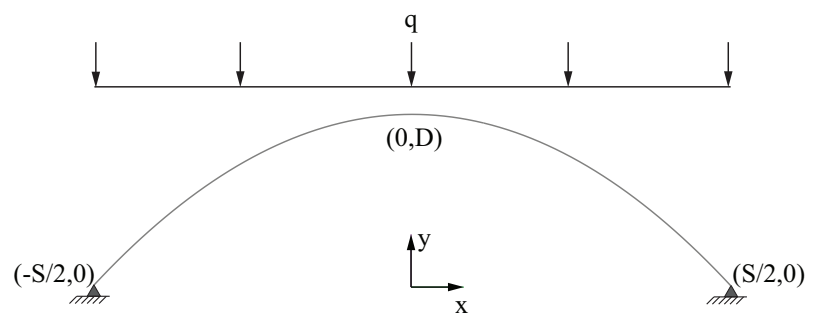

Figure 7: Geometric coordinates and loading. 


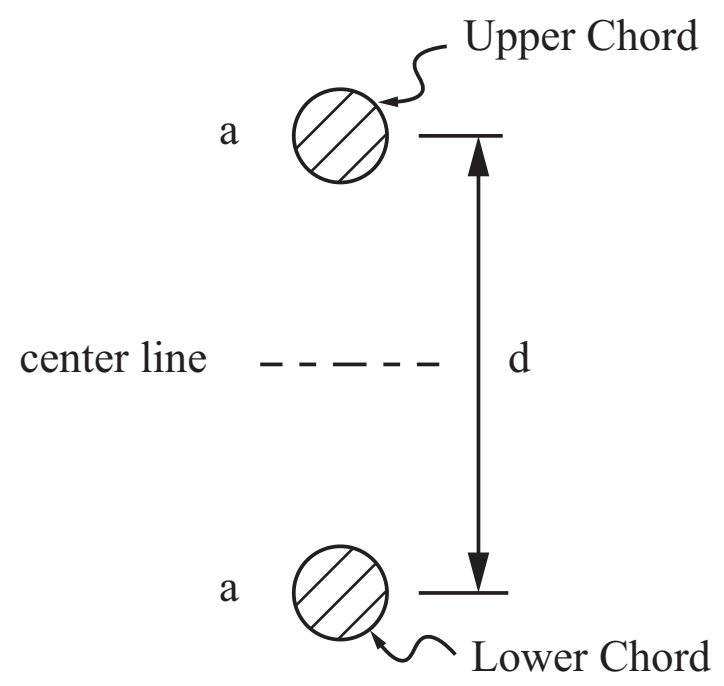

Figure 8: Arch cross-section. 


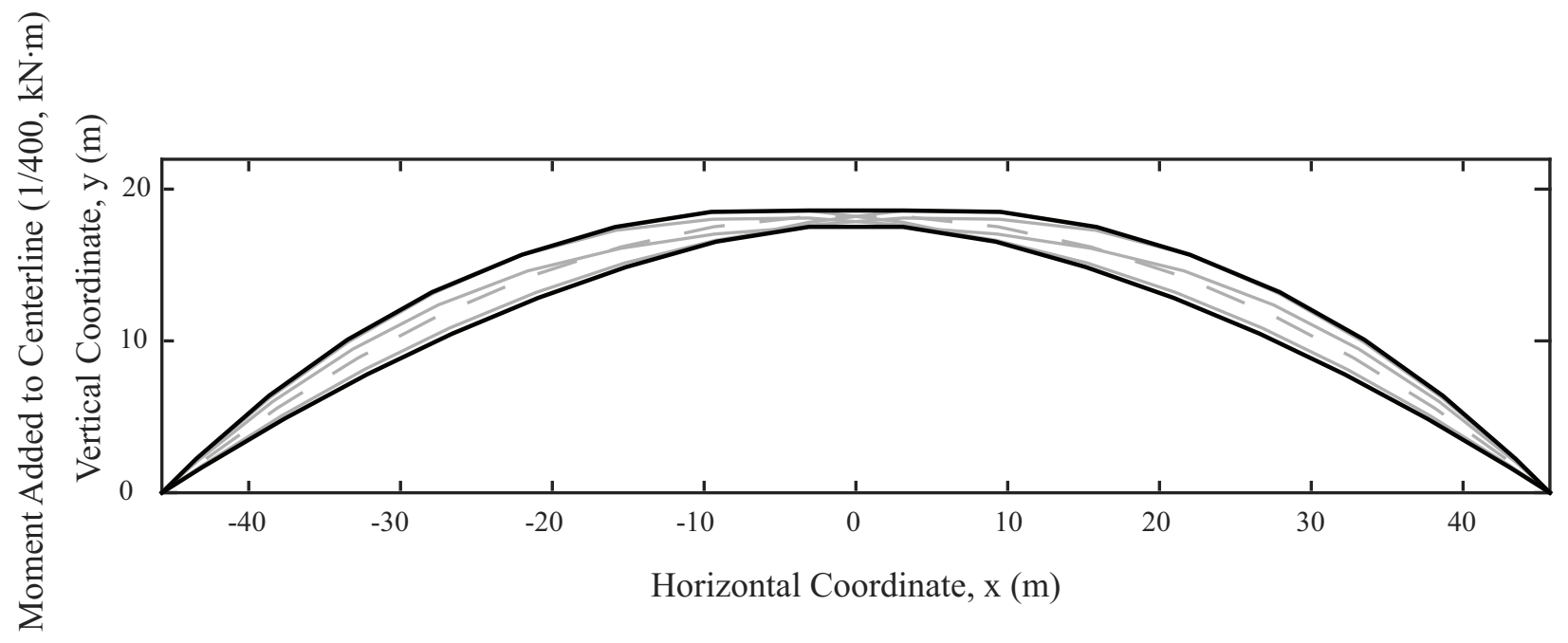

Figure 9: Moment envelope for $91.4 \mathrm{~m} \mathrm{(300} \mathrm{ft)} \mathrm{two-hinged} \mathrm{arch} \mathrm{overlayed} \mathrm{over} \mathrm{the} \mathrm{arch} \mathrm{centerline} \mathrm{(dashed}$ line). Envelope is shown in black. Individual moment for different load combinations in gray. 


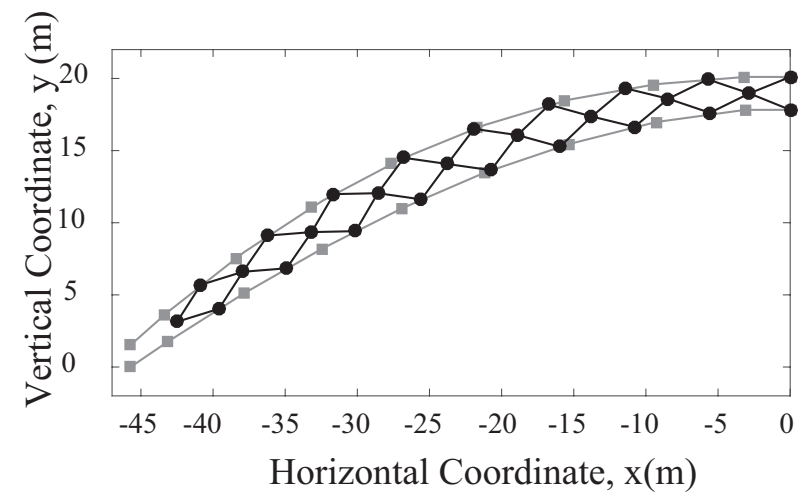

Figure 10: Form development for the variable depth two-hinged arch. Gray square markers indicate the discrete points at which the depth was calculated. The polynomial curves fit to these points are shown as gray lines. The module links are shown as black lines with the revolute joints as black circles. Shown for

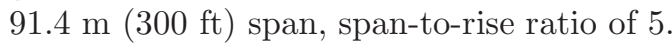



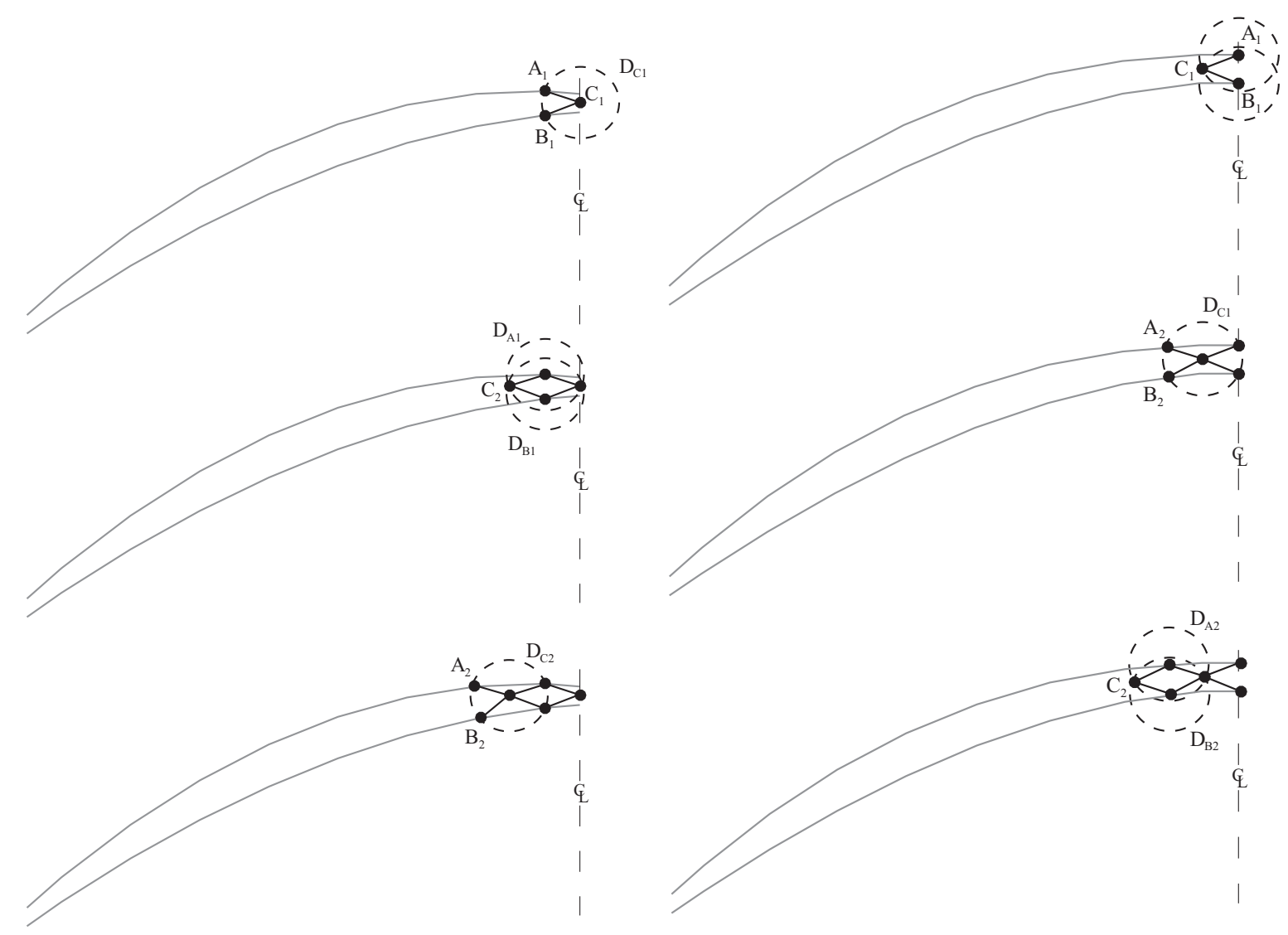

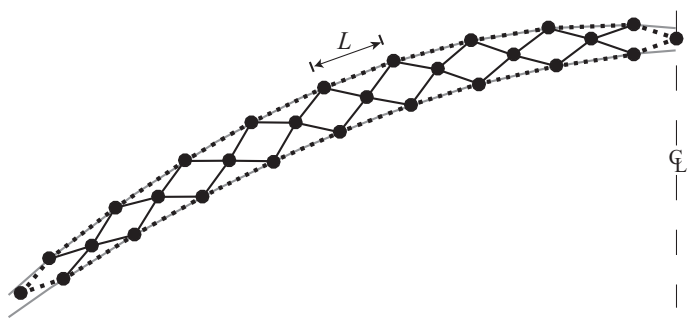

(A)

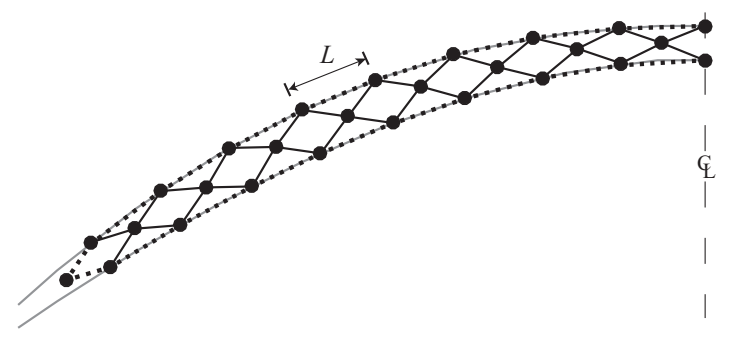

(B)

Figure 11: Scribing procedure for (A) three-hinged arch, and (B) two-hinged arch. Gray lines indicate the upper and lower continuous curves. Black circles indicate points $A, B$, and $C$. Dashed lines show the circles of radius $l$ used for scribing (indicated by letter $D$ ). Dotted lines on bottom images indicate upper and lower chords of the final arches. Dimension $L$ and $l$ are indicated. 


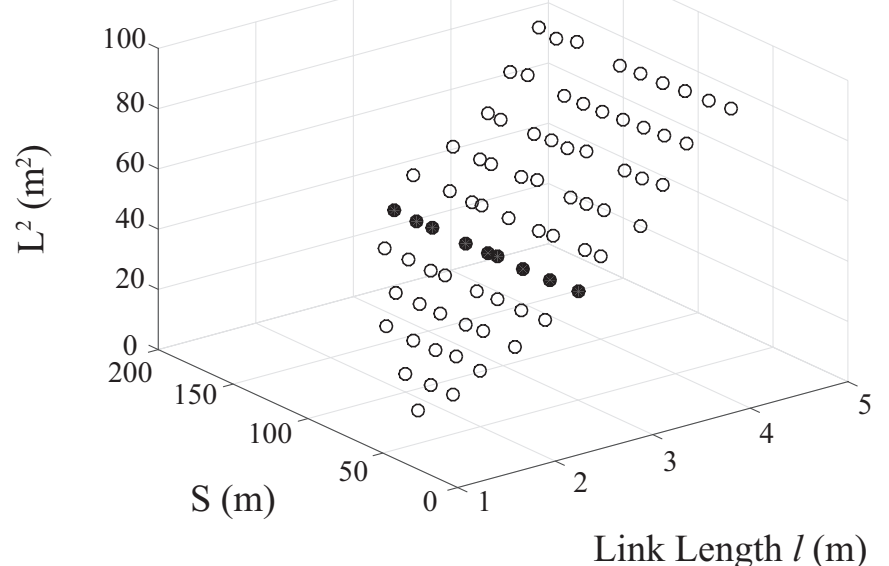

Figure 12: Results of parametric study to determine link length for the three-hinged arch. $3.05 \mathrm{~m} \mathrm{(10} \mathrm{ft)}$ link length is highlighted in black. 


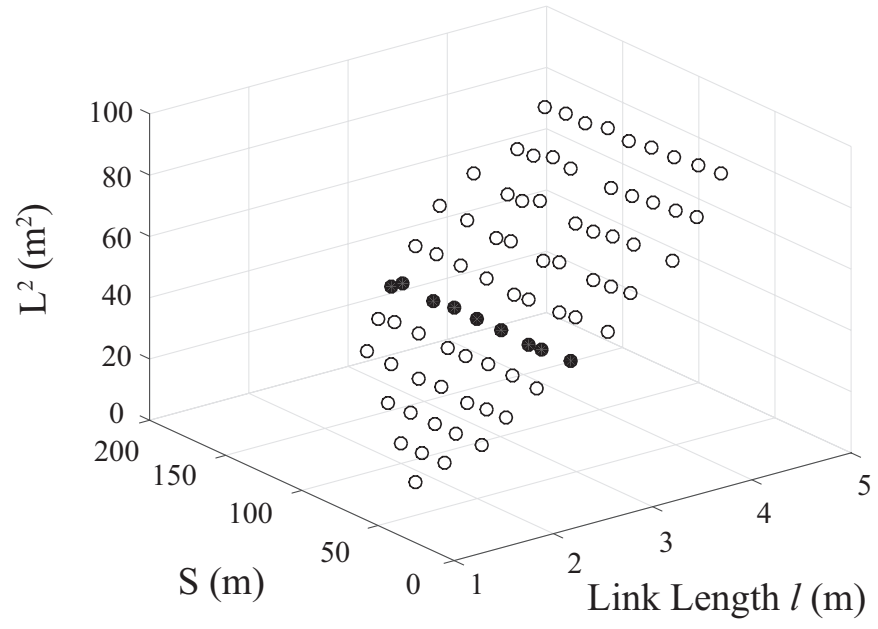

Figure 13: Results of parametric study to determine link length for the two-hinged arch. $3.05 \mathrm{~m}$ (10 ft) link length is highlighted in black. 

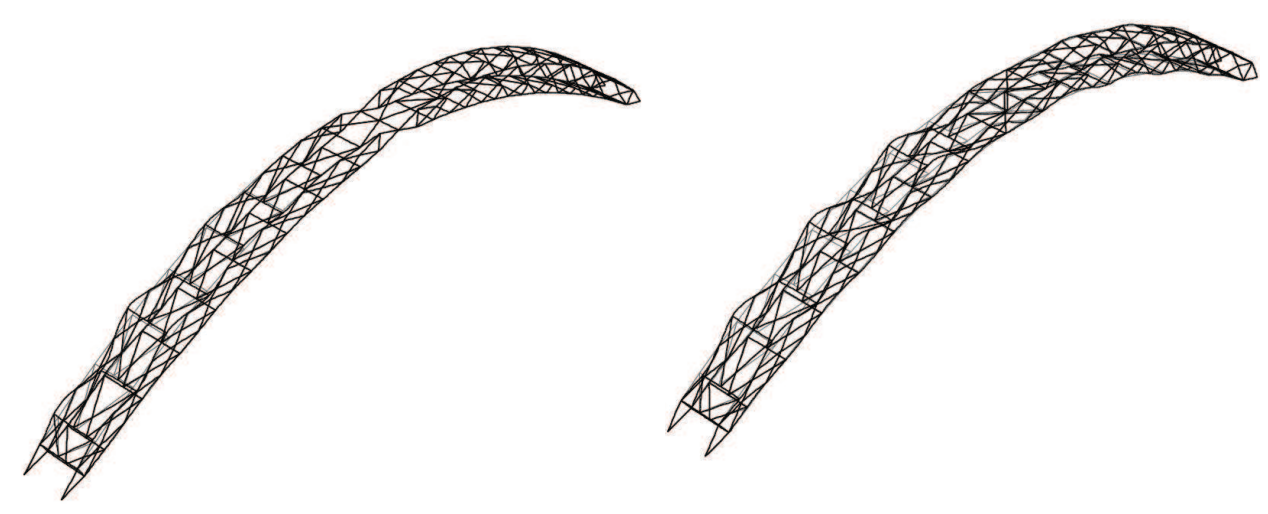

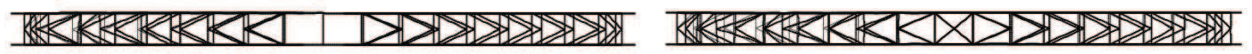

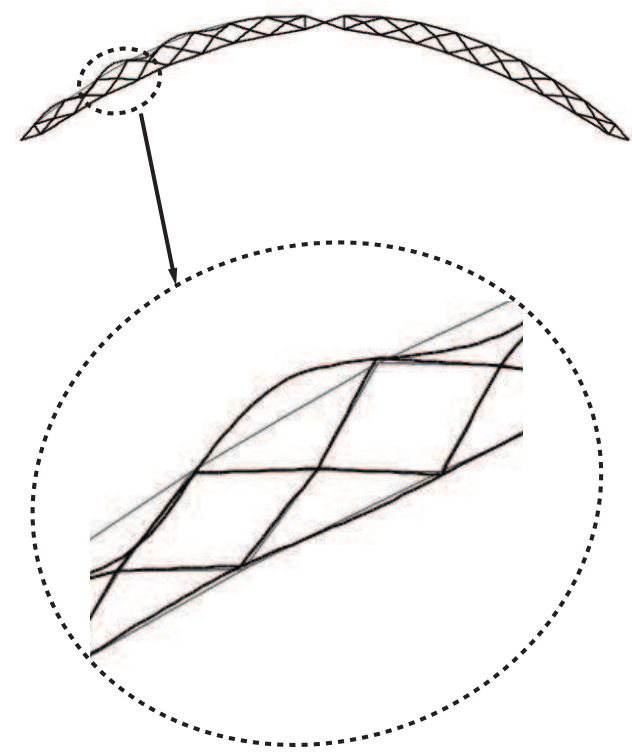

(A)

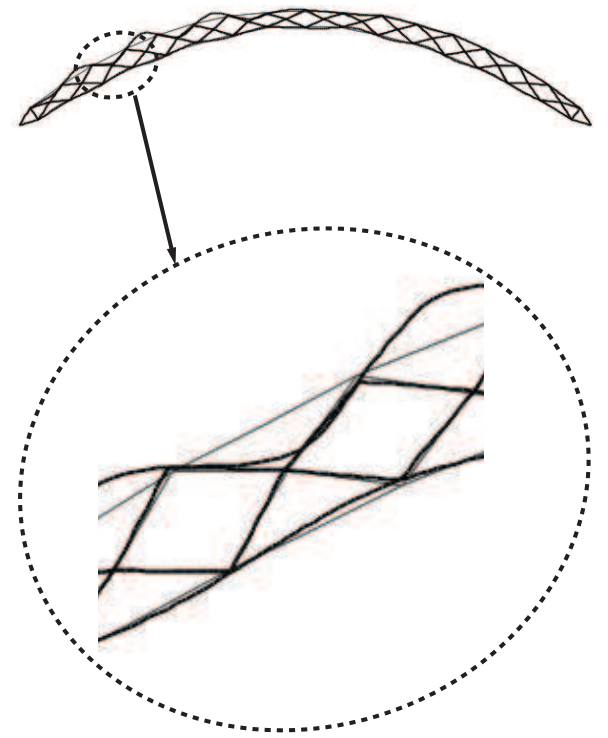

(B)

Figure 14: Buckled shape for (A) three-hinged arch buckling mode under dead load, live load over left half span, and wind load (Buckling Factor $=2.71$ ), and $(B)$ two-hinged arch buckling mode under dead load, live load over left half span, and wind load (Buckling Factor $=3.09$ ). The top row indicates the isometric view, the second row indicates the plan view, the third row indicates the elevation view, and the fourth row focuses on the buckled shape of one portion of the structure in elevation view. 
[1] Russell, B.R., Thrall, A.P.. Portable and rapidly deployable bridges: Historical perspective and recent technology developments. Journal of Bridge Engineering 2013;18(10):1074-1085.

[2] Joiner, J.H.. One More River to Cross: The Story of British Military Bridging. South Yorkshire, UK: Pen and Sword Books Ltd; 2001.

[3] Gerbo, E.J., Casias, C.M., Thrall, A.P., Zoli, T.P.. New bridge forms composed of modular bridge panels. Journal of Bridge Engineering 2015;In press.

[4] AASHTO, . AASHTO LRFD Bridge Design Specifications, Customary U.S. Units. 6th edition ed.; Washington, D.C.: American Association of State Highway and Transportation Officials (AASHTO); 2012.

[5] Todisco, L., Corres Peiretti, H., Mueller, C.. Funicularity through external posttensioning: Design philosophy and computational tool. Journal of Structural Engineering 2016;142(2):04015141.

[6] Akbarzadeh, M., Van Mele, T., Block, P.. On the equilibrium of funicular polyhedral frames and convex polyhedral force diagrams. Computer-Aided Design 2015;6:118-128.

[7] Beghini, L.L., Carrion, J., Beghini, A., Mazurek, A., Baker, W.F.. Structural optimization using graphic statics. Structural and Multidisciplinary Optimization 2014;49(3):351-366.

[8] AUTODESK, . AutoCAD. 2016. <http://www.autodesk.com/products/autocad/overview> (May 30, 2016).

[9] Baker, W.F., Beghini, L.L., Mazurek, A., Carrion, J., Beghini, A.. Maxwell's reciprocal diagrams and discrete michell frames. Structural and Multidisciplinary Optimization 2013;48(2):267-277.

[10] Allen, E., Zalewski, W.. Form and Forces: Desiging Efficient and Expressive Structures. New York, NY: Wiley; 2009.

[11] Zalewski, W., Allen, E.. Shaping Structures: Statics. New York, NY: Wiley; 1998.

[12] Winter, G., Urquhart, L.C., O'Rourke, C.E., Nilson, A.. Design of Concrete Structures. New York, NY: McGraw-Hill Book Company; 1964.

[13] Nettleton, D.A.. Arch bridges. 1977. Bridge Division, Office of Engineering, Federal Highway Administration, U.S. Department of Transportation, Washington D.C.

[14] AISC, . Steel Construction Maunal. 14th edition ed.; Chicago, IL: American Institute of Steel Construction (AISC); 2011.

[15] Hartenberg, R.S., Denavit, J.. Kinematic Synthesis of Linkages. New York, NY: McGraw-Hill Book Company; 1964. 
[16] Euler, L.. Mechanica, Sive, Motus Scientia Analytice exposita. St. Petersburg, Russia: Petropoli: Ex Typographia Academiae Scientiarum; 1736.

[17] Waddell, J.. Bridge Engineering. New York, NY: John Wily \& Sons, Inc; 1916.

[18] Thrall, A.P., Adriaenssens, S., Paya-Zaforteza, I., Zoli, T.P.. Linkage-based movable bridges: Design methodology and three novel forms. Engineering Structures 2012;37:214-223.

[19] Ario, I., Nakazawa, M., Tanaka, Y., Tanikura, I., Ono, S.. Development of a prototype deployable bridge based on origami skill. Automation in Construction 2013;32:104-111.

[20] Clevett, M.L., Lowdermilk, R.H., Bright, J.K., Coulter, C.S., Melton, T.W.. Expandable portable bridge structure. 1986. U.S. Patent 4,628,560. U.S. Patent and Trademark Office, Washington, DC.

[21] Computers and Structures, Inc., . SAP2000: Integrated software for structural analysis and design. 2016. <http://www.csiamerica.com/products/sap2000> (January 16, 2016).

[22] Department of the Army, . Bailey bridge. In: Field Manual No. 5-277. Washington, DC: Headquarters, Department of the Army; 1986,. 